\title{
The genetic structure of populations of the vulnerable aquatic macrophyte Ranunculus nipponicus (Ranunculaceae)
}

\author{
Keiichi Koga $\cdot$ Yasuro Kadono $\cdot$ Hiroaki Setoguchi
}

Published online: 26 April 2007

C) The Botanical Society of Japan and Springer 2007

Erratum to: J Plant Res 120:167-174

DOI 10.1007/s10265-006-0060-1

In Table 1, in the column headed "Sample size $(N)$ ", an error was inadvertently introduced during correction by the publisher. The second value (upstream sample size in Hirukuchi) should be "(42)", not '(4v2)".

The online version of the original article can be found at http://dx.doi.org/10.1007/s10265-006-0060-1.

K. Koga $\cdot$ H. Setoguchi $(\bowtie)$

Department of Biology,

Graduate School of Human and Environmental Studies,

Kyoto University, Kyoto 606-8501, Japan

e-mail: seto@botany.mbox.media.kyoto-u.ac.jp

Y. Kadono

Department of Biology, Faculty of Science,

Kobe University, Kobe 657-8501, Japan 